- Oral midazolam sedation $(0.5 \mathrm{mg} / \mathrm{kg})$ appears to be an acceptable form of sedation for 10-16-year-old paediatric dental patients

- Oral midazolam may provide a safe alternative to nitrous oxide/oxygen sedation and general anaesthesia.

- Oral midazolam sedation must only be used in accordance with General Dental Council Guidelines by an experienced dental sedationist.

\title{
A study of the effectiveness of oral midazolam sedation for orthodontic extraction of permanent teeth in children: a prospective, randomised, controlled, crossover trial
}

\author{
K. E. Wilson, ${ }^{1}$ R. R. Welbury ${ }^{2}$ and N. M. Girdler ${ }^{3}$
}

\begin{abstract}
Objectives To assess the safety, effectiveness and acceptability of oral midazolam sedation for orthodontic extraction of permanent teeth in children.

Design A prospective, randomised, controlled, crossover trial. Methods A total of 26 children aged $10-16$ (ASA I), referred for orthodontic extraction of premolar or canine teeth under sedation, were included in the study. Each child required two treatment sessions for the extraction of equivalent teeth on opposite sides of the mouth. Each subject was sedated with either oral midazolam $(0.5 \mathrm{mg} / \mathrm{kg})$ or nitrous oxide and oxygen (30\%/70\%) at the first visit and the alternative form at the second visit. At each visit two teeth were extracted, one upper and one lower. Heart rate, arterial oxygen saturation, respiration rate, sedation and behavioural scores were recorded every five minutes. Overall behaviour, patient acceptance and patient satisfaction were recorded at the end of treatment.

Results Of the 26 children included in the study there were 12 males and 14 females. The mean age was 12.5 years. The mean heart rate and respiratory rate for both groups were similar and within acceptable clinical limits. The lowest mean arterial oxygen saturation levels for nitrous oxide and midazolam sedation were $97.7 \%$ and $95.0 \%$ respectively. Although midazolam caused greater oxygen desaturation, the range $(91 \%$ - 100\%) was within safe limits for conscious sedation. The mean level of sedation was greater in the midazolam group compared with the nitrous oxide group and all but one case completed treatment. A total of 23 patients (88\%) said they would be prepared to have oral midazolam sedation again and 17 (65\%) actually preferred oral midazolam to nitrous oxide sedation.

Conclusion Oral midazolam $(0.5 \mathrm{mg} / \mathrm{kg})$ appears to be a safe and acceptable form of sedation for $10-16$ year old paediatric dental patients.
\end{abstract}

\footnotetext{
${ }^{1}$ Honorary Staff Grade, Department of Sedation, Newcastle Dental School and Hospital, ${ }^{2}$ Professor of Paediatric Dentistry, Glasgow Dental School and Hospital, ${ }^{3}$ Consultant/Senior Lecturer, Department of Sedation, Newcastle Dental School and Hospital,

*Correspondence to: Dr Kathy Wilson, Department of Sedation, Newcastle Dental School Framlington Place, Newcastle upon Tyne, NE2 4BW

Email:katherine.wilson@ncl.ac.uk
}

\section{Refereed paper}

Received 01.03.01; Accepted 15.01.02

(๑) British Dental Journal 2002; 192: 457-462
Conscious sedation is used extensively in dentistry to help anxious patients undergo dental treatment with minimal physiological and psychological stress. The General Dental Council ${ }^{1}$ and the Royal College of Anaesthetists ${ }^{2}$ have both encouraged the use of conscious sedation as a safe alternative to general anaesthesia for dental care. The main type of sedation employed for paediatric dental patients is an inhalational technique which uses titrated doses of nitrous oxide and oxygen known as relative analgesia sedation. ${ }^{3}$ The technique has a proven safety record and is the mainstay of paediatric dental sedation in the UK. ${ }^{4-7}$

The favourable properties of nitrous oxide, plus the absence of any systemic metabolism makes it an almost ideal sedation agent. However nitrous oxide does present a potential hazard to clinical staff who are involved in providing inhalational sedation on a regular basis. ${ }^{8,9}$ There is evidence that chronic exposure to nitrous oxide gas can produce haematological, reproductive and neurological problems. ${ }^{10-13}$ Although the use of scavenging equipment, good technique and well-ventilated surgeries has significantly reduced the level of staff exposure, there is inevitably some residual surgery pollution. ${ }^{9,14}$ The Health Services Advisory Committee have recently addressed the issue of occupational hazards and have recommended occupational exposure levels of staff to nitrous oxide and other anaesthetic gases. ${ }^{15}$ Although the literature suggests there is minimal risk to personnel carrying out conscious sedation with nitrous oxide and oxygen, provided scavenging, good technique and good ventilation are used, there is concern relating to high occupational exposure and some experts predict that nitrous oxide may ultimately be banned as an anaesthetic sedation agent in order to completely eliminate the risk to clinical personnel. ${ }^{12,16}$

In light of the potential problems associated with the use of nitrous oxide and the need for alternative management options to general anaesthesia, it is important to seek alternative sedation drugs for use in paediatric dental patients. The use of oral sedation techniques may provide a useful alternative. Oral premedication with benzodiazepines is already in widespread use in medicine for paediatric patients. ${ }^{17,18}$ Midazolam, the benzodiazepine with the most favourable pharmacological profile, has been found to be an effective oral premedicament prior to anaesthesia for surgical procedures. ${ }^{19,20,21}$ Midazolam is not licensed for use as a sedative in 
children in the UK and has received limited evaluation for this purpose in this country. In other countries oral midazolam sedation has mainly been tested in combination with other sedation agents, but such polypharmacy is not recommended for use in UK dental practice. ${ }^{22,23}$ Other benzodiazepines, such as temazepam and alternative sedatives including the antihistamines, do not have such favourable pharmacological profiles as midazolam when used in children. ${ }^{24}$ The relatively short half-life of midazolam produces rapid onset and recovery and is well suited for ambulatory patients in dental practice. ${ }^{25,26}$

It has been suggested that oral midazolam sedation could provide an effective method of conscious sedation in child dental patients. The aim of the study was to evaluate the safety, effectiveness and patient acceptability of oral midazolam sedation in paediatric dental patients undergoing orthodontic extractions of permanent teeth, in comparison with nitrous oxide and oxygen sedation.

\section{METHODS}

\section{Study design}

The study was designed as a prospective, randomised, controlled, crossover trial and had the approval of Newcastle and North Tyneside Local Ethics Committee. A total of 26 patients were recruited who were: aged 10-16 years, ASA I and II and referred to the Sedation Department at Newcastle Dental Hospital for orthodontic extraction of at least four teeth (premolars or canines) under local anaesthetic and sedation. Each required bilateral, identical extractions on opposite sides of the mouth. A full verbal and written explanation of the study was given to the patient and the parent and informed written consent was gained for the treatment and inclusion in the study. Midazolam is licensed for use only by the intravenous route, as the study involved an unlicensed route of administration, parental consent was obtained.

Each patient was allocated randomly to receive either oral midazolam sedation or nitrous oxide sedation at their first appointment. The alternative technique was used at their second appointment. Nitrous oxide sedation acted as control, being the standard paediatric sedation technique in current use in the UK. Dental extractions were carried out on opposite sides of the mouth at consecutive appointments, the order (right or left) being allocated randomly.

\section{Clinical technique}

An experienced operator / sedationist (a dentist trained in sedation techniques and holding the University of Newcastle Diploma in Conscious Sedation), working under the supervision of a consultant in dental sedation, was responsible for administering the sedation. The dental sedationist also monitored the patient's clinical status throughout each session, assisted by an appropriately trained dental sedation nurse. All patients were instructed to starve for two hours prior to their treatment appointment.

Inhalation Sedation: A Quantiflex MDM relative analgesia machine was used to administer nitrous oxide via a nasal mask. The nitrous oxide was titrated in $10 \%$ increments to a final dose of 30\% nitrous oxide, 70\% oxygen, whilst the clinician provided reassurance and positive reinforcement. The authors realise that nitrous oxide is normally titrated to the maximum desired level for individual patients. However for the purposes of the study a maximum dose of 30\% nitrous oxide was used as a control. Studies have shown that levels of $20 \%-30 \%$ nitrous oxide provide an adequate level of sedation, without the risk of side effects of over sedation. ${ }^{27}$ In addition an audit carried out in the Department of Sedation at Newcastle Dental School showed that the median level of nitrous oxide used in 500 patients was 30\% (unpublished audit).
Once the level of 30\% nitrous oxide had been reached it was continued throughout the subsequent dental treatment. Upon completion of treatment, the nitrous oxide flow was switched off and 100\% oxygen administered for 2 minutes before the nasal mask was removed.

Oral midazolam sedation: Midazolam was administered orally at a dose of $0.5 \mathrm{mg} / \mathrm{kg}$. This dose has been shown to produce good anxiolysis when used as premedication prior to anaesthesia..$^{21,22,24}$ As midazolam has a bitter taste it was mixed with $30 \mathrm{ml}$ of sugar-free orange cordial in water. The drug was administered 45 minutes before treatment to ensure the patient was adequately sedated prior to treatment being carried out, a time period used by other researchers. ${ }^{28}$ The patient was then monitored in a recovery area under the supervision of their parent and a sedation nurse.

Monitoring: Patients were monitored clinically and by pulse oximetry throughout the period of sedation, dental treatment and recovery. Clinical signs which were monitored included pulse, respiration rate, colour and level of responsiveness.

Dental Treatment: 20\% benzocaine topical anaesthetic was applied to the gingivae for 2 minutes and then 2\% lidocaine with 1:80,000 epinephrine local analgesic was administered in a standard technique for each quadrant eg upper premolar or canine: buccal infiltration and palatal infiltration via the buccal papilla; lower premolar or canine: buccal infiltration followed by lingual infiltration via the buccal papilla. Once analgesia had been achieved the two teeth were extracted.

\section{Recovery and discharge}

Once dental treatment was complete the patient was transferred to recovery and allowed to recover for at least 20 minutes where they were supervised by their parent and a sedation nurse. At the end of that period of time the operator assessed the patient's fitness for discharge. Full written and verbal postoperative sedation and surgical instructions were provided.

\section{Assessment criteria}

The following parameters were measured in order to evaluate the technique:

Physiological status: Baseline weight, blood pressure, pulse, respiration rate and oxygen saturation were recorded immediately prior to the administration of sedation. Once the sedative had been administered the pulse, respiration rate, and oxygen saturation were monitored continuously throughout treatment and the was data recorded every 5 minutes. The blood pressure was reassessed if there was any concern over the patient's clinical condition.

\begin{tabular}{ll} 
Table 1 Briekopf and Buttner - Classification of emotional status \\
\hline Score & Description \\
\hline 1 & Irritated: Awake, restless, crying \\
2 & Normal: Awake, calm \\
3 & Inactive: Tired, hardly moving \\
4 & Sleepy: Drowsy, without reaction but rousable
\end{tabular}

Table 2 Frankl Behaviour Rating Score

\begin{tabular}{ll}
\hline Score & Description \\
\hline 1 & Refusal/Distress \\
2 & Uncooperative/Reluctant \\
3 & Co-operative/Reserved \\
4 & Interested/Enjoyed
\end{tabular}


Table 3 Houpt Behaviour Rating Scale

\begin{tabular}{ll}
\hline Score & Description \\
\hline 1 & Aborted: No treatment rendered \\
2 & Poor: Treatment interrupted, only partial treatment was completed \\
3 & Fair: Treatment interrupted but eventually completed \\
4 & Good: Difficult but all treatment was performed \\
5 & Very Good: Some limited crying or movement \\
6 & Excellent: No crying or movement
\end{tabular}

Level of sedation and emotional status: The sedation level was recorded every 5 minutes using the classification of emotional status designed by Brietkopf and Buttner ${ }^{29}$ (Table 1).

Behaviour during treatment. The Frankl Behaviour Rating Scale $^{30}$ was used to grade the child's behaviour during treatment (Table 2).

Outcome of treatment: The Houpt Behaviour Rating Scale ${ }^{30}$ was used to record the overall behaviour and outcome of treatment (Table 3).

Patient Preference: After each session the patient was given a questionnaire to be completed at home. The questionnaire asked the child to grade how well they liked the sedation, how they felt about the sedation and, following the second appointment, which type of sedation they preferred. The parent was also asked to record post-operative adverse effects and their perception of how well the child coped with each type of sedation.

\section{Data analysis}

Data was entered onto a PC database and analysed using Minitab ${ }^{\circledR}$ software. Measurements of physiological status, emotion, and behaviour all have scales consisting of ordinal or nominal data and they were analysed using paired $t$-test, Mann Whitney $U$ tests or Chi squared tests as appropriate. Data from the patient satisfaction questionnaire was transcribed for qualitative data and paired $t$-test used for quantitative data.

\section{RESULTS}

Twenty six subjects in total were included in the study, the mean age being 12.5 years (range 10-16 years); 12 were male and 14 female and all were classified as ASA I.

\section{Physiological status}

The mean lowest arterial oxygen saturation recorded was 97.7\% for the nitrous oxide group and 95.0\% for the midazolam group. The difference in the values was found to be statistically signifi- cant $(\mathrm{P}<0.0005)$, however the range of values for the midazolam group was within acceptable and safe limits for conscious sedation $(91 \%-100 \%)$. The mean respiration rate for the nitrous oxide group was 14.8 breaths per minute and for the midazolam group 14.6 breaths per minute. The difference was not found to be statistically significant $(\mathrm{P}>0.5)$.

\section{Sedation, treatment and recovery times}

The mean time to maximum level of sedation for the nitrous oxide group was 5.2 minutes, compared with 26.8 minutes for the midazolam group. The difference was found to be highly significant $(p<0.0001)$. However there was no significant difference in the maximum duration of dental treatment ie from administration of local analgesic to completion of dental extractions between the two groups, with a mean of 10 minutes for the nitrous oxide group and 10.8 minutes for the midazolam group. The maximum time spent in recovery (time from the completion of treatment) was significantly different between the two groups, with mean values of 20 and 39.7 minutes for nitrous oxide and midazolam respectively The difference was found to be significant at $\mathrm{P}<0.0005$. The total appointment time (time from entering surgery to discharge home) was significantly greater for the midazolam group $(\mathrm{p}<0.0005)$, with the mean total appointment times being 32.8 minutes and 93.6 minutes for the nitrous oxide and midazolam groups respectively. The overall results are illustrated in Figure 1.

\section{Maximum level of sedation and emotional status}

In the nitrous oxide group the maximum sedation score was 2 (awake and calm) for twenty-four children and 3 (tired, hardly moving) for two children. In the midazolam group seven children had a maximum sedation score of 2 , thirteen children a maximum score of 3 and four scored 4 (drowsy, without reaction but rousable) (Figure 2). Chi squared test indicated the difference between the nitrous oxide and midazolam groups to be highly significant $(\mathrm{P}<0.0001)$.

\section{Behaviour during treatment}

Behaviour scores during treatment are clearly illustrated in Figure 3 and show that there was no difference in the overall behaviour scores between the two sedation groups.

\section{Overall behaviour / Outcome of treatment}

The Houpt scale was used to record overall behaviour and outcome of treatment. In the nitrous oxide group 21 children scored 6 - excellent, three scored 5 - very good, one scored 4 good and one child scored 1-treatment aborted. In the midazolam group 18 scored 6-excellent, three scored 5-very good, two scored 4-good, two scored 3-fair, and one child scored 1 treatment aborted. The difference in the two groups was not significant $(\mathrm{P}>0.5)$.
Figure 1 Duration of different stages of the appointment. The time periods are: Sedation: time to reach the maximum level of sedation; Dental treatment: the maximum time for the dental treatment to be carried out: Recovery: the maximum time spent in recovery and Total appointment: total appointment time from the start of sedation to discharge.

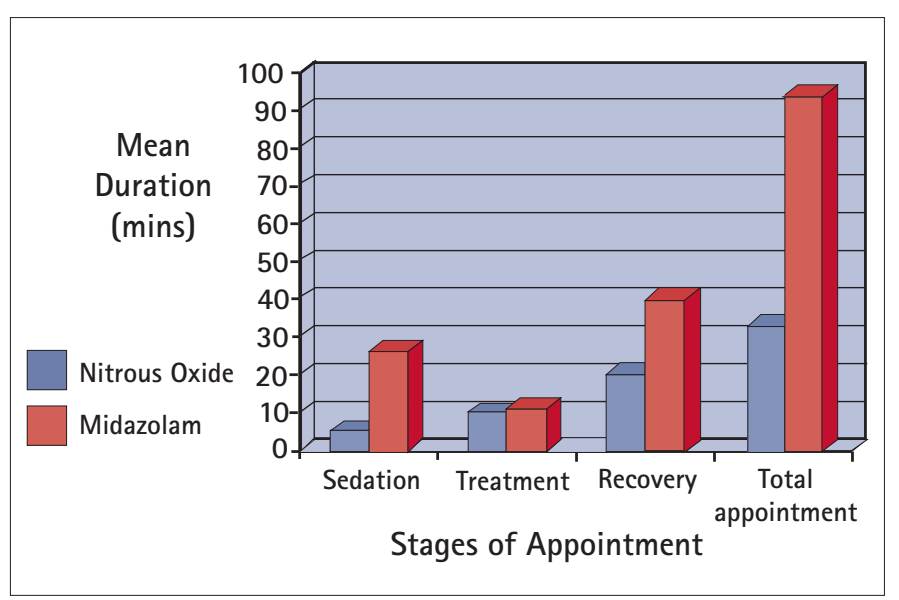




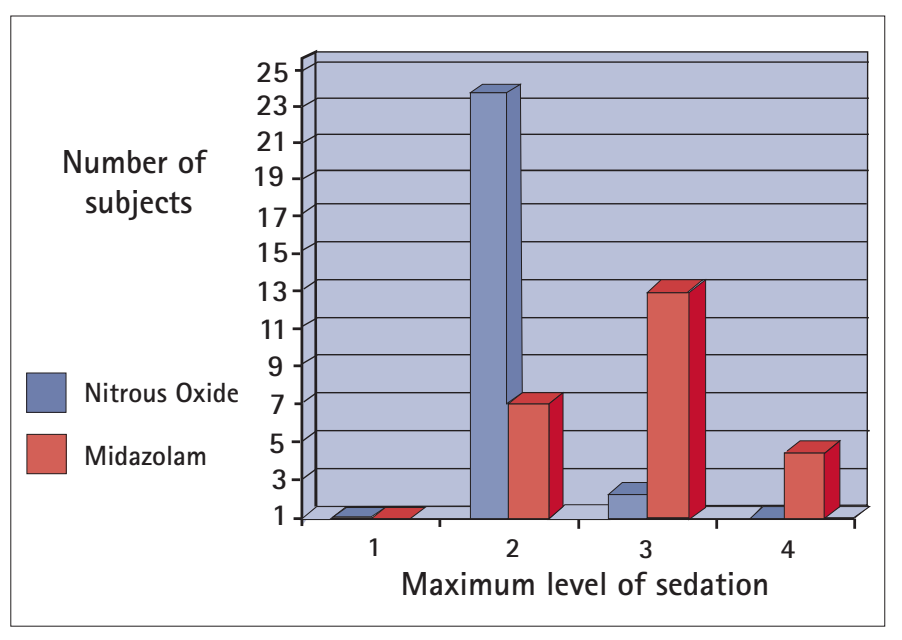

The patient's opinion of the treatment

Twelve children in the midazolam group felt very relaxed compared with the five in the nitrous oxide group. Seven children reported feeling relaxed with midazolam and eight felt relaxed with nitrous oxide. Four children felt normal with midazolam and five felt normal with nitrous oxide. Of those reporting feeling anxious two were in the midazolam groups and seven in the nitrous oxide group. Only one child in each sedation group reported feeling very anxious. These differences were not found to be statistically significant $(\mathrm{P}>0.5)$.

\section{Recall of the treatment}

The patients were asked if they could remember having the local anaesthetic, extraction of the teeth and being in recovery. In the nitrous oxide group 23 children could remember having the local anaesthetic compared with six in the midazolam group. This difference was highly significant at $\mathrm{P}<0.0001$. In the nitrous oxide group 20 patients remembered having their teeth extracted compared with 11 in the midazolam group $(\mathrm{P}<$ 0.05). Recall of the time spent in recovery was not significantly different between the two groups; 22 children in the nitrous oxide group and 14 children in the midazolam group remembered this time (Figure 4).

\section{Preference for sedation technique}

All subjects were asked if they would be prepared to have the same form of sedation again and which method they preferred. Twenty children (77\%) said they would have nitrous oxide again and 23 (88\%) said they would have midazolam again. Seventeen subjects (65\%) preferred midazolam sedation and 9 (35\%) preferred nitrous oxide sedation.

\section{DISCUSSION}

Recent government guidelines have stressed the importance of the use of alternative forms of pain and anxiety control for

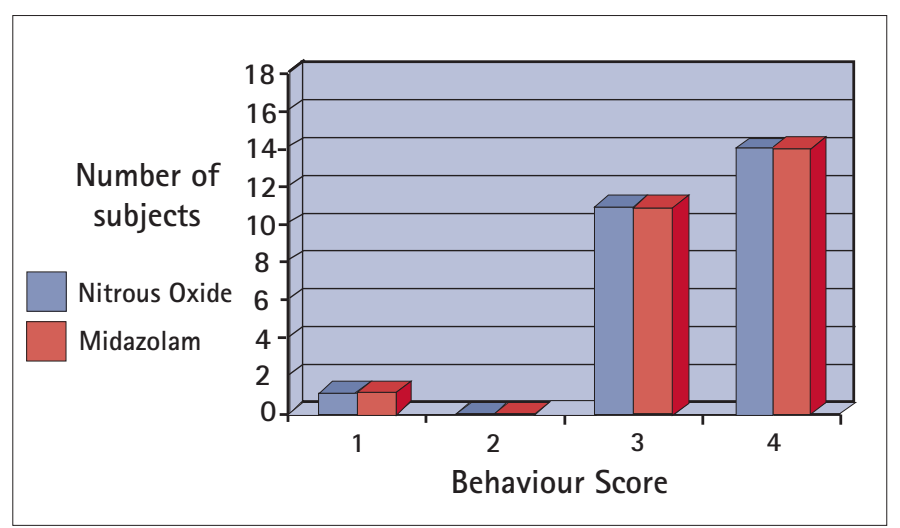

Figure 2 Maximum levels of sedation. Using the Brietkopf and Buttner classification of emotional status the results are represented as the number of subjects scoring a maximum score of; 1 (awake, restless), 2 (awake, calm), 3 (Tired, hardly moving), 4 (drowsy, without reaction hardly moving).

dental treatment in place of general anaesthesia where appropriate. ${ }^{31}$ Such alternatives include the use of different conscious sedation techniques. Relative analgesia sedation with nitrous oxide is the mainstay of conscious sedation services for children receiving dental treatment in the UK. However evidence suggests that nitrous oxide may pose a potential risk to clinical personnel who are providing this form of sedation on a regular basis. ${ }^{8-13}$ Indeed in the future nitrous oxide may be banned altogether for medical use. ${ }^{12,16}$ All paediatric patients may not accept nitrous oxide sedation and at present there is no alternative. It is for these reasons that research into other sedation techniques for paediatric dental patients is important.

Benzodiazepines are used extensively in adult patients but little work has been carried out in the UK to consider the use of oral midazolam in children. This study was designed to assess the safety and effectiveness of oral midazolam as an alternative to nitrous oxide sedation in paediatric dental patients. The study was a randomised crossover design, but owing to the nature of the different sedation techniques could not be a blind trial.

All 26 patients who took part in the study were healthy and in the age range 10 - 16 years. The treatment required by all subjects was equivalent orthodontic extractions of at least four equivalent permanent teeth, in opposite sides of the mouth. The assessment criteria included physiological status, treatment and recovery times, levels of sedation, behaviour and acceptance of treatment, the patient's opinion of the treatment, recall of the treatment and preference for sedation technique.

The mean lowest oxygen saturation recorded for each group varied slightly, 97.7\% for the nitrous oxide group and 95.0\% for the midazolam group. The difference was found to be statistically significant, however from a clinical point of view the saturation readings for the midazolam group were within safe and acceptable limits with a range of 91-100\%. In the clinical setting the minimum accepted saturation level is $90 \%$ and at no time was this level recorded. The mean respiratory rate for both

Figure 3 Behaviour scores during treatment. Using the Frankl Behaviour Rating Scale the results are presented as the number of subjects scoring; 1 (refusal/distress), 2 (uncooperative/reluctant), 3 (co-operative/reserved), 4 (interested/enjoyed). 
Figure 4 Positive recall of the appointment. The results are presented as the number of subjects who remembered having a local anaesthetic, having teeth extracted and being in recovery.

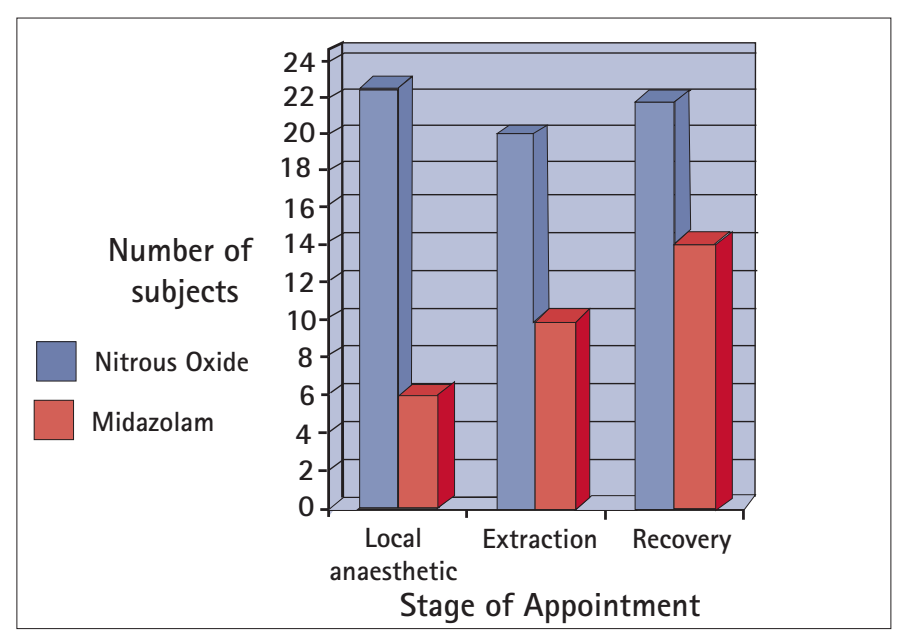

reported high behaviour scores throughout treatment. Only one treatment session with midazolam in the current study had to be aborted due to the patient experiencing dis-inhibition, where he became very restless and un-cooperative. Paradoxical reactions to midazolam have been reported by other researchers, ${ }^{19,28}$ but are uncommon. However, one of the main reasons for the limited use of midazolam in children is due to the unpredictable effects that it can produce. This study supports evidence from other studies that such adverse effects of midazolam occur but are not common and should not deter its possible use for paediatric dental sedation.

As would be expected due to the anterograde amnesic effects of midazolam, fewer patients could recall the different elements of their treatment. Only 23\% of patients could remember having local anaesthetic and the extractions following sedation with midazolam. This can be especially beneficial when unpleasant procedures such as extractions are being carried out. This amnesic effect did not seem to cause any distress among the subjects, as it was not reported on any of the patient evaluation forms as being a problem. Indeed only $19 \%$ of patients in both sedation groups reported any side effects and these included sleepiness, dizziness and slight headache, none of which were reported as being of major concern to the child or parent.

The patient's opinion of the sedation techniques is important to evaluate, it provides an indication of their experience and feelings towards the care they have received. When children received midazolam only two found it unpleasant or very unpleasant. More children felt very relaxed with midazolam than nitrous oxide. Midazolam would appear to produce a pleasant experience for the patients. Of the two sedation techniques experienced, 23 (88\%) patients said they would be prepared to have midazolam sedation again and 17 (65\%) said they preferred midazolam to nitrous oxide sedation.

The results of the study are promising with regard to the use of oral midazolam $(0.5 \mathrm{mg} / \mathrm{kg})$ for conscious sedation in paediatric dental patients aged $10-16$. Midazolam is a licensed drug for use by the intravenous route only. However, the use of licensed drugs for unlicensed applications is acceptable in paediatric practice where there is no suitable alternative, provided the consent of the parent has been obtained. ${ }^{33}$ The prescribing practitioner must also choose the medicine, which offers benefits to the child, and in the case of midazolam there is evidence to suggest that oral midazolam is a useful anxiolytic in paediatric patients. ${ }^{17,19,20}$

The safety of the patient is paramount when providing conscious sedation and in the case of oral midazolam sedation it is mandatory to monitor the patient's arterial oxygen saturation with a pulse oximeter at all times. It is also recommended that the patient's blood pressure be monitored throughout treatment and recovery; a dedicated trained sedation nurse is essential to carry out and record all monitoring. 
As oral midazolam cannot be titrated to the individual's response, anyone undertaking this technique must be competent in managing the over-sedated patient. This would include the ability to cannulate a patient, should the reversal agent flumazenil be required. It is also essential that the operator/sedationist be proficient in delivering paediatric life support in the event of an emergency.

In conclusion this study has shown that midazolam appears to be a safe sedation agent for use in $10-16$ year old healthy patients, producing no significant physiological changes and few side effects. Only one case of paradoxical reaction was reported, but otherwise it appears to be well accepted by patients and produces good anxiolysis, which enables treatment to be carried out effectively. Oral midazolam produces significant amnesic effects, which may be of benefit, particularly with very anxious children who may be undergoing their first experience of extractions. Most patients would accept treatment under midazolam sedation again and the majority preferred it to nitrous oxide sedation. When considering the use of oral midazolam sedation, it must be carried out in accordance with General Dental Council Guidlines. ${ }^{1}$

1. General Dental Council. Maintaining standards: Guidance to dentists on professional and personal conduct. London: General Dental Council, 1999.

2. Royal College of Anaesthetists. Standards and guidelines for general anaesthesia for dentistry. London: Royal College of Anaesthetists, 1999.

3. Roberts $\mathrm{GJ}$ J. Inhalational sedation (relative analgesia) with oxygen/nitrous oxide gas mixture: principles. Dental Update 1990; 17: 139-146.

4. Crawford A N. The use of nitrous oxide/oxygen inhalational sedation with local analgesia as an alternative to general anaesthetic in children. Br Dent J 1990; 168: 395-398.

5. Shaw A J, Meechan J G, Kilpatrick N M, Welbury R R. The use of inhalation sedation and local anaesthesia instead of general anaesthesia for extractions and minor oral surgery in children: a prospective study. Int J Paed Dent 1996; 6: 7-11.

6. Blain K M, Hill FJ. The use of inhalational sedation and local analgesia as an alternative to general anaesthesia for dental extractions in children. Br Dent J 1998; 184: 608-611.

7. Shepherd A R, Hill FJ. Orthodontic extractions: a comparative study of inhalation sedation and general anaesthesia. Br Dent J 2000; 188: 329-331.

8. Donaldson D, Meechan J G. The hazards of chronic exposure to nitrous oxide: an update. BrDent J 1995; 178: 95-100.

9. Henderson K A, Matthews I P. Environmental monitoring of nitrous oxide during dental anaesthesia. Br Dent J 2000; 188: 617-619.

10. Yagiela J A. Health hazards and nitrous oxide: A time for reappraisal. Anaesthesia
Progress 1991; 38: 1-11.

11. Starch D J. Nitrous oxide sedation: understanding the benefits and risks. Am J Dent 1995; 8: 47-50.

12. Shaw A D, Morgan M. Nitrous oxide: time to stop laughing. Anaesthesia 1998; 53 : 213-215.

13. Girdler N M, Hill C M. Sedation in Dentistry. Oxford: Butterworth Heinemann,1998.

14. Girdler N M, Stirling P A. Investigation of nitrous oxide pollution arising from inhalational sedation for the extraction of teeth in child patients. Int J Paed Dent 1998; 8: 93-102.

15. Health and Safety Executive. Anaesthetic Agents: Controlling exposure under COSHH. London: HMSO, 1995.

16. Henderson KA, Matthews I P. Environmental monitoring of nitrous oxide during dental anaesthesia. BrDent J 2000; 188 : 617-619.

17. Parnis S J, Foate J A, Van der Walt J H. Oral midazolam is an effective pre-medication for children having day stay anaesthesia. Anaesthesia Intensive Care 1992; 20: 9-14.

18. Elder J S, Longnecker R. Pre-medication with oral midazolam for voiding cystourethrography in children. Am J Roengeneology 1995; 5: 1229-1232.

19. McMillan C O, Spahr-Schopfer I A, Sikich N, Hartley E, Lerman J. Premedication of children with oral midazolam Can J Anaes 1992; 39: 545-550.

20. Ong B C, Ng A S, Chw S L. Oral pre-medication in paediatric day surgery. Singapore Med J 1996; 37: 139-142.

21. Riva J, Pap M, Lauber C. Oral pre-medication with midazolam in paediatric anaesthesia: Effects on sedation and gastric content. Paed Anaes 1997; 7: 191-196.

22. Hartgraves P M, Primosch R E. An evaluation of oral and nasal midazolam for paediatric dental sedation. J Dent Child 1994; 61: 175-181.

23. Poswillo D E. Report of an expert working party on general anaesthesia, sedation and resuscitation in dentistry. London: Department of Health, 1990.

24. Skelly A M, Girdler N M, File S. The use of temazepam elixir in surgical dental sedation: A comparison with intravenous midazolam. BrDent J 1992; 172: 153.

25. Reeves I G, Fragen R J, Vinik H R. Midazolam: Pharmacology and uses. Anaesthesiology 1985; 62:310-324.

26. Pyne K, Mattheyse FJ, Leibenberg D. Pharmacokinetics of midazolam in paediatric patients. European J Clin Pharm 1989; 37: 267-272.

27. Cooper J, Jobling D, Edmunds D H. Sedation for minor oral surgery: inhalation sedation with 25 per cent nitrous oxide. J Dent 1978; 6: 265-267.

28. Rodrigo M R, Cheung LK. Oral midazolam sedation in third molar surgery. Int J Oral Maxillofac Surg 1987; 16: 333-337.

29. Krafft T C, Kramer N, Kunzelmann K, Hickel R. Experience with midazolam as sedative in the dental treatment of uncooperative children. J Dent Child 1993; 295- 299.

30. Hosey M T, Blinkhorn AS. An evaluation of four methods of assessing the behaviour of anxious child dental patients. Int J Paed Dent. 1995; 5: 87-95.

31. A Conscious Decision: A review of the use of general anaesthesia and conscious sedation in primary dental care. London: Dept of Health, 2000.

32. Shapira J, Gideon $H_{1}$ Eyal B et al. The effectiveness of midazolam and hydroxyzine as sedative agents for young pediatric dental patients. J Dent Child 1996; 421-425.

33. Policy statement produced by the joint RCPCH/NPPG Standing Committee on Medicines. The use of unlicensed medicines or licensed medicines for unlicensed applications in paediatric practice. London: Royal College of Paediatrics and Child Health, 2000 